\title{
A Trade-off Model for Green Supply Chain Design: An Efficiency-versus-Emission Analysis
}

\author{
$\mathrm{Hao} \mathrm{Yu}$ \\ Department of Industrial Engineering \\ Faculty of Engineering Science and Technology \\ UiT-The Arctic University of Norway \\ Narvik, Norway \\ e-mail: hao.yu@uit.no
}

\author{
Wei Deng Solvang \\ Department of Industrial Engineering \\ Faculty of Engineering Science and Technology \\ UiT-The Arctic University of Norway \\ Narvik, Norway \\ e-mail: wei.d.solvang@uit.no
}

\begin{abstract}
With the growing concern of climate change and global warming, the reduction on carbon emissions of supply chain activities have been increasingly focused by both academics and corporates in the last two decades. The increased public awareness on sustainability and stringent environmental policies together enforce the companies to re-think their practices in supply chain design and operations in an efficient while environmentally friendly fashion. This paper proposes a mathematical programming model for green supply chain design taking into consideration of both efficiency and carbonemissions. First, a bi-objective MILP model is formulated for green supply chain design. Second, both weighting method and augmented $\varepsilon$-constrained method are introduced for resolving the trade-off between the two objective functions. Third, through the numerical experiment, the quality and computational efficiency of the non-dominant Pareto optimal solutions calculated by both weighing method and augmented $\varepsilon$ constraint method are compared, and the result is discussed latter in this paper.
\end{abstract}

Keywords-Green supply chain; optimization; network design; constraint method; Pareto optimal solution

\section{INTRODUCTION}

The concept of supply chain management has been raised and extensively focused for over three decades. A typical supply chain includes several actors, i.e., raw material supplier, producer, distributor, wholesaler, retailer and customer [1]. Each actor adds value to the products and/or process and affects the material, information and cash flows of the supply chain. Due to this reason, the optimal design and planning of supply chain structure and operations are of great importance for companies to maintaining a sound efficiency, competitiveness and sustainability.

The design of a supply chain structure and network is one of the most important decision-making problems in supply chain management [2], and it usually involves both strategic and operational decisions in order to determine the nodes (facility locations) and arcs (material flows and/or vehicle routes) in a supply chain network. Traditionally, the main focus of supply chain design and operation management is to manage an effective material flow while maintaining cost efficiency $[3,4]$. In recent years, with the growing concern of climate change and global warming, the reduction on carbon emissions of supply chain activities have been increasingly focused by both academics and corporates in literature. The concepts of green supply chain and sustainable supply chain are proposed to address this problem. The definition of green supply chain has been given by many researchers [5], and the main focus is to achieve an optimal break-even point at which the efficiency and environmental impact of a supply chain, usually quantified and measured by carbon emissions [6-8], is balanced through decision-making at strategic, tactical and operational levels.

The incorporation of "green thinking" in supply chain design usually affects the supply chain efficiency and profitability in a negative manner $[4,6,7]$. Thus, an optimal trade-off or Pareto optimal solution is desired and is worthy to be investigated. In order to provide a thorough understanding of the trade-off between efficiency and environmental impact in supply chain design, significant efforts have been invested in previous literature for developing advanced tools and decision methods. Comprehensive literature surveys of green supply chain design are given by several researchers with focuses on conceptual development [9], quantitative methods [10], mathematical optimization models and approaches [11, 12].

This paper investigates some recent publications with the focus on both methodological development and practices of green supply chain design. In order to balance the trade-off among cost efficiency, environmental impact and lead time, Boonsothonsatit et al. [13] develop a fuzzy goal programming with weighted min-max operator to determine the supply chain configuration. Coskun et al. [14] formulate a goal programming model for green supply chain design with the consideration of customer segment. The model aims at maximizing the overall utility of a supply chain through compensating the environmental influence. Through the use of two questionnaires and a fuzzy multi-criteria decision model, Diabat et al. [15] investigate the performance and practices of green supply chain management in the automotive industry. Yu and Solvang [16] formulate a goal programming for balancing the efficiency and carbon emission of green supply chain design.

Jin et al. [17] formulates and investigates the influence of implementing three different policies on carbon control (carbon cap, carbon tariff, and cap-and-trade) on supply chain 
design. Zhou et al. [8] formulate a mathematical optimization model for green supply chain design with focus on the implementation of carbon tariff policy. Taking into account of economic, environmental and social sustainability, Miret et al. [18] develop a multi-objective MILP model solved with goal programming approach for the planning of a bioethanol green supply chain. In this paper, the environmental impact is quantified by life cycle analysis, and the social indicator is measured by energy-food ratio and the number of jobs created by the supply chain. Wang et al. [4] investigate the Pareto optimal trade-off between efficiency and environmental influence of green supply chain design.

Govindan et al. [19] propose a bi-objective mathematical model for decision support of both green supply chain design and sustainable order allocation, and the model is resolved with hybrid method incorporating both adapted multiobjective variable neighborhood search and adapted multiobjective electromagnetism mechanism algorithm. The integration of supplier selection with green supply chain design is also focused by Ameknassi et al. [20]. In their paper, a stochastic multi-objective mathematical programming is formulated and is resolved with $\varepsilon$-constraint method for designing a multi-product multi-period green supply chain structure. Govindan et al. [21] investigate a bi-objective location routing problem for the design and operation management of a perishable food supply chain, and the model aim at finding out the optimal trade-off between efficiency and environmental impact.

Taking into account of the reverse direction of supply chain management for the value recapture from recycling and recovery of used merchandise, $\mathrm{Yu}$ and Solvang [21] propose a bi-objective MILP model for simultaneously minimizing the cost and carbon emission in the planning of a reverse logistics system. Considering the inexactness and uncertainty related to the input information of the decision model, Feito-Cespon et al. [22] develop a stochastic programming model for the redesign and re-optimization of a sustainable reverse supply chain. The model aims to determine the optimal trade-off among economic, environmental and social issues related to the reverse supply chain. Yu and Solvang [23] and $\mathrm{Yu}$ and Solvang [7] formulate stochastic MILP models incorporating with soft and hard constraints on carbon emission requirement for sustainable design of a reverse supply chain. The result from the research works illustrates the influence of implementing two different carbon policies, namely, carbon tariff and carbon cap on sustainable reverse supply chain design under market fluctuation.

Compared with previous research works in green supply chain design, the current paper focuses on the decision support through providing decision makers with a set of non-dominant optimal efficiency-versus-emission trade-offs for posteriori decision-making. In order to solve the trade-off problem, a MILP model is first formulated in Section II. Section III introduces the augmented $\varepsilon$-constraint method as well as its advantages in determining the non-dominant optimal tradeoffs among several conflicting objectives comparing with traditional $\varepsilon$-constraint method and the common scalarization method. Section IV validates the model and compares the results calculated with different solution methods. Section V provides the conclusion and future suggestions.

\section{MATHEMATICAL MODEL FOR GREEN SUPPLY CHAIN DESIGN WITH EFFICIENCY-VERSUS-EMISSION ANALYSIS}

Figure 1 presents a supply chain structure with four echelons including supplier, production plant, distribution center and customer. The supply chain design focuses on the optimal efficiency-versus-emission trade-offs through the decision-making on both strategic and tactical levels.

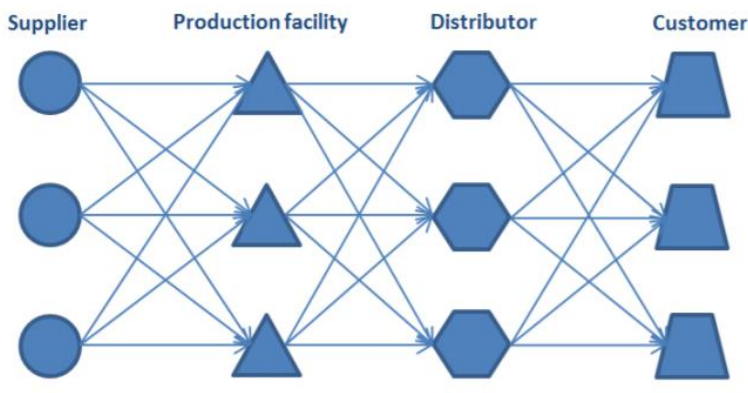

Figure 1. Supply chain structure [16].

In order to model the green supply chain design problem, common assumptions are made [6, 19, 24]:

- The parameters are deterministic and known.

- The planning is given on a single-period basis.

- The material flow in the supply chain is featured with single product.

- The material flow among nodes in the same echelon is prohibited.

A. Sets

The sets of the nodes in the supply chain network is first defined.

$S \quad$ Set of suppliers, indexed by $S$

$P \quad$ Set of candidates for production plant, indexed by $p$

$D$ Set of candidates for distribution center, indexed by $d$

$C$ Set of customers, indexed by $c$

\section{B. Decision Variables}

The decisions on the locations of production plants and distribution centers and the allocation of customer demands will be made in each optimal trade-off scenario.

$\emptyset_{p} \quad$ Binary decision variable for production plant

$\emptyset_{d} \quad$ Binary decision variable for distribution center

$F l_{s p} \quad$ Material flow between nodes $s$ and $p$

$F l_{p d} \quad$ Material flow between nodes $p$ and $d$

$F l_{d c} \quad$ Material flow between nodes $d$ and $c$

\section{Parameters}

The parameters of the decision model are given as follows. 
This manuscript is a preprint version and final version is available on IEEE Xplore.

Doi: 10.1109/ICITM.2018.8333934

$Q_{p} \quad$ Opening cost of production plant

$Q_{d} \quad$ Opening cost of distribution center

$G_{p} \quad$ Operation cost per unit of production plant

$G_{d} \quad$ Operation cost per unit of distribution center

$\rho_{s p} \quad$ Transport cost per unit between nodes $s$ and $p$

$\rho_{p d} \quad$ Transport cost per unit between nodes $p$ and $d$

$\rho_{d c} \quad$ Transport cost per unit between nodes $d$ and $c$

$C O E_{p}$ Carbon emission per unit at production plant

$C O E_{d}$ Carbon emission per unit at distribution center

$E_{s p}$ Carbon emission per unit transport between nodes $s$ and $p$

$E_{p d}$ Carbon emission per unit transport between nodes $p$ and $d$

$E_{d c}$ Carbon emission per unit transport between nodes $d$ and $c$

$\omega_{p} \quad$ Capacity of production plant

$\omega_{d} \quad$ Capacity of distribution center

$\operatorname{Dem}_{c} \quad$ Customer demand

$\beta_{d}$ Conversion ratio between raw materials and finished productions

$\mu_{p} \quad$ Utilization ratio for fulfilling custom orders at distribution centers

\section{Objective Functions}

The MILP model includes two objective functions given in Eqs. (1) and (2). Eq. (1) optimizes the efficiency through minimizing the setup and operation costs of the supply chain. Eq. (2) reduces the environmental influence by minimizing the carbon emission.

$$
\begin{aligned}
\operatorname{Min} z(1)=\sum_{p \in P} \phi_{p}\left(Q_{p}+G_{p} \sum_{s \in S} F l_{s p}\right) & +\sum_{d \in D} \emptyset_{d}\left(Q_{d}+G_{d} \sum_{p \in P} F l_{p d}\right) \\
& +\sum_{s \in S} \sum_{p \in P} F l_{s p} \rho_{s p}+\sum_{p \in P} \sum_{d \in D} F l_{p d} \rho_{p d} \\
& +\sum_{d \in D} \sum_{c \in C} F l_{d c} \rho_{d c} \\
\operatorname{Min} z(2)=\sum_{s \in S} \sum_{p \in P} \operatorname{COE} E_{p} F l_{s p}+\sum_{p \in P} \sum_{d \in D} C O E_{d} F l_{p d} & +\sum_{s \in S} \sum_{p \in P} F l_{s p} E_{s p}+\sum_{p \in P} \sum_{d \in D} F l_{p d} E_{p d} \\
& +\sum_{d \in D} \sum_{c \in C} F l_{d c} E_{d c}
\end{aligned}
$$

\section{E. Requirements of Decision Variables}

Eqs. (3) and (4) show the integer and non-negative requirements of the decision variables.

$$
\begin{gathered}
\emptyset_{p}, \emptyset_{d} \in\{0,1\} \\
F l_{s p}, F l_{p d}, F l_{d c} \geq 0
\end{gathered}
$$

\section{F. Capacity limitation}

Eqs. (5) and (6) give the capacity limitation of production plants and distribution centers.

$$
\begin{aligned}
& \sum_{S \in S} F l_{s p} \leq \omega_{p}, \forall p \in P \\
& \sum_{p \in P} F l_{p d} \leq \omega_{d}, \forall d \in D
\end{aligned}
$$

\section{G. Flow Restriction}

Eqs. (7)-(11) are flow restrictions of the supply chain. Eq. (7) ensures the custom demands are met. Eqs. (8) and (9) specifies the input and output ratio at production plants and distribution centers. Herein, it is noted the input products at distribution centers may not be totally allocated to satisfy the customer due to the limitation of operation and transportation, otherwise, $\beta_{d}$ equals to 1 . Eqs. (10) and (11) establish the requirements of the existence of material flow between different nodes in the supply chain network.

$$
\begin{gathered}
\sum_{d \in D} F l_{d c}=D e m_{c}, \forall c \in C \\
\beta_{d} \sum_{p \in P} F l_{p d}=\sum_{c \in C} F l_{d c}, \forall d \in D \\
\mu_{p} \sum_{s \in S} F l_{s p}=\sum_{d \in D} F l_{p d}, \forall p \in P \\
F l_{s p} F l_{p d} \leq \emptyset_{p} U, \forall s \in S, \forall p \in P, \forall d \in D \\
F l_{p d} F l_{d c} \leq \emptyset_{d} U, \forall p \in P, \forall d \in D, \forall c \in C
\end{gathered}
$$

\section{SOLUTION METHOD}

The mathematical model formulated in this paper is a multi-objective MILP model, which aims at determining the optimal efficiency-versus-emission trade-off in green supply chain design. Considering the characteristics of a supply chain design problem with multiple conflicting objectives, the mathematical model can be given in a more general form with the help of the concepts of vector and matrix, as shown in Eq. (12).

$$
\begin{aligned}
& \operatorname{Min} z_{1}(\vec{x}, \vec{y})=\overrightarrow{a_{1}} \cdot \vec{x}+\boldsymbol{A}_{\mathbf{1}} \cdot \vec{y} \\
& \operatorname{Min} z_{2}(\vec{x}, \vec{y})=\overrightarrow{a_{2}} \cdot \vec{x}+\boldsymbol{A}_{\mathbf{2}} \cdot \vec{y} \\
& \cdots \\
& \text { Sin } z_{x}(\vec{x}, \vec{y})=\overrightarrow{a_{x}} \cdot \vec{x}+\boldsymbol{A}_{\boldsymbol{x}} \cdot \vec{y} \\
& \\
& \vec{x} \in\{0,1\} \\
& \vec{y} \geq 0 \\
& \boldsymbol{C} \cdot \vec{y} \leq \boldsymbol{D} \cdot \vec{x} \\
& \boldsymbol{E} \cdot \vec{y}=\boldsymbol{F}
\end{aligned}
$$

Eq. (12) formulates a general MILP model for supply chain design with objectives $\left\{z_{1}(\vec{x}, \vec{y}), z_{2}(\vec{x}, \vec{y}), \ldots, z_{x}(\vec{x}, \vec{y})\right\}$, and it provides decision makers with the optimal decisions on facility locations with integer variable vector $\vec{x}$ and allocations of material follow among different facilities with continuous variable vector $\vec{y}$. The input parameters are given in 
vectors $\left\{\overrightarrow{a_{1}}, \overrightarrow{a_{2}}, \ldots, \overrightarrow{a_{x}}\right\}$ and matrixes $\left\{\boldsymbol{A}_{1}, \boldsymbol{A}_{2}, \ldots, \boldsymbol{A}_{x}, \boldsymbol{C}, \boldsymbol{D}, \boldsymbol{E}, \boldsymbol{F}\right\}$. In addition, it is noteworthy that Eqs. (5) and (6) and Eqs. (10) and (11) in the MILP model are aggregated in the general form.

In order to find out the optimal trade-offs among several conflicting objectives in supply chain design, a break-even point must be determined, and it is called Pareto optimality in operational research. The definition of Pareto optimality has been given by many authors [21, 25], based on the general model formulated above, a solution vector $\left(\overrightarrow{x_{o}}, \overrightarrow{y_{o}}\right)$ is considered to be a Pareto optimality of the multi-objective programming if and only if it is impossible to find out another solution vector with $z_{k}(\vec{x}, \vec{y}) \leq z_{k}\left(\overrightarrow{x_{o}}, \overrightarrow{y_{o}}\right)$ for $k=1,2, \ldots, x$ and $z_{i}(\vec{x}, \vec{y}) \neq z_{i}\left(\overrightarrow{x_{o}}, \overrightarrow{y_{o}}\right)$ for at least one $i$. Thus, it is obvious that a Pareto optimality of a multi-objective programming is the optimal trade-off among several conflicting objective functions, and the performance of one objective at the Pareto optimality cannot be improved without compensating the performance of the other objectives. Furthermore, it is obviously that an infinite set of Pareto optimality are existed in a multi-objective programming problem.

The most commonly used method to find out the Pareto optimality of a multi-objective programming problem is scalarization method including weighted sum method and constraint method $[25,26]$.

\section{A. Weighted sum method}

Due to its simplicity and effectiveness in resolving the trade-offs of multi-objective programming problems particularly the problems for priori decision-making [27], weighted sum method has been extensively used in literature [6]. With the weighted sum method, the importance of each objective function is first determined by the decision-makers and given as the weight, and then the objective functions can be aggregated through the summation of the weighted objective functions. Eventually, the problem becomes a single-objective MILP optimization and can be easily solved. Herein, it is noteworthy that the measures of each objective value may not be identical in some decision-making problems (e.g. the efficiency and emission in green supply chain design), and in those cases, the objective functions will first be normalized before they can be summed up.

$$
\begin{gathered}
\vec{x} \in\{0,1\} \\
\vec{y} \geq 0 \\
\boldsymbol{C} \cdot \vec{y} \leq \boldsymbol{D} \cdot \vec{x} \\
\boldsymbol{E} \cdot \vec{y}=\boldsymbol{F}
\end{gathered}
$$$$
\operatorname{Min} w_{1} z_{1}(\vec{x}, \vec{y})+w_{2} z_{2}(\vec{x}, \vec{y}), \ldots, w_{x} z_{x}(\vec{x}, \vec{y})
$$

Even though weighted sum method has been widely used in resolving complex multicriteria decision-making problems, it do has some weaknesses [28]. First, the determination of the weights has to be done before the decision-making, which means decision-makers must have a very clear understanding of the importance of each objective, and this could be very difficult at the very beginning or sometimes even impossible. Second, when the weighted sum method is used to generate a set of Pareto optimality, it is not able to generate a full set of Pareto optimality. Furthermore, with the weighted sum method, the allocation of the Pareto optimality is not evenly distributed even if evenly distributed weights combination is used.

\section{B. Constraint method}

Constraint method is another very commonly used method to find out the Pareto optimality of a multi-objective optimization problem. The basic idea of constraint method is to take one objective function to optimize while converting the other objective functions into inequality constraints with an upper bound $\varepsilon_{k}$ for $k=1,2, \ldots, x$, as illustrated in Eq. (14). Due to the introduction of the upper bound, it is alternatively named $\varepsilon$-constraint method.

$$
\begin{gathered}
\text { Min } z_{1}(\vec{x}, \vec{y})=\overrightarrow{a_{1}} \cdot \vec{x}+\boldsymbol{A}_{\mathbf{1}} \cdot \vec{y} \\
z_{2}(\vec{x}, \vec{y}) \leq \varepsilon_{2} \\
\cdots \ldots \\
z_{x}(\vec{x}, \vec{y}) \leq \varepsilon_{x} \\
\vec{x} \in\{0,1\} \\
\vec{y} \geq 0 \\
\boldsymbol{C} \cdot \vec{y} \leq \boldsymbol{D} \cdot \vec{x} \\
\boldsymbol{E} \cdot \vec{y}=\boldsymbol{F}
\end{gathered}
$$

The $\varepsilon$-constraint method can effectively resolve the problems of the weighted sum method and can generate a set of evenly distributed Pareto optimality without the input on the importance of each objective from decision-maker. Nevertheless, there are weaknesses of the traditional $\varepsilon$ constraint method related to the selection of the values of $\varepsilon$, and this may lead to dominant or weak solutions as discussed by Mavrotas [29]. Compare with the definition of Pareto optimality, a solution vector $\left(\overrightarrow{x_{w}}, \overrightarrow{y_{w}}\right)$ is considered to be a dominant or weak Pareto optimality of the multi-objective programming if and only if it is impossible to find out another solution vector with $z_{k}(\vec{x}, \vec{y}) \leq z_{k}\left(\overrightarrow{x_{w}}, \overrightarrow{y_{w}}\right)$ for $k=1,2, \ldots, x$. Thus, it is obvious that the set of Pareto optimality is subset of weak Pareto optimality.

$$
\begin{aligned}
& \operatorname{Min} z_{1}(\vec{x}, \vec{y})=\overrightarrow{a_{1}} \cdot \vec{x}+\boldsymbol{A}_{\mathbf{1}} \cdot \vec{y}+\text { eps } \times\left(s_{2}+, \ldots,+s_{x}\right) \\
& \qquad \begin{array}{c}
\text { S.t. } \\
z_{2}(\vec{x}, \vec{y})+s_{2}=\varepsilon_{2} \\
\cdots \cdots \\
z_{x}(\vec{x}, \vec{y})+s_{x}=\varepsilon_{x} \\
\vec{x} \in\{0,1\} \\
\vec{y} \geq 0 \\
\boldsymbol{C} \cdot \vec{y} \leq \boldsymbol{D} \cdot \vec{x} \\
\boldsymbol{E} \cdot \vec{y}=\boldsymbol{F}
\end{array}
\end{aligned}
$$

In order to determine a set of non-dominant Pareto optimality, Mavrotas [29] develops an augmented $\varepsilon$ constraint method. First, the payoff matrix is calculated by a lexicographical method through the continuous optimization in order to find out the non-dominant payoff matrix. From the calculation of payoff matrix, the range of the solution area can 
This manuscript is a preprint version and final version is available on IEEE Xplore.

be divided in order to determine the benchmark value of each $\varepsilon$. The second improvement made in the augmented $\varepsilon$ constraint method is to convert the inequality constraints to equality constraints through the introduction of slack as shown in Eq. (15), where eps is a small enough number between $10^{-3}$ and $10^{-6}$ [29]. This change guarantees the efficient Pareto optimality through binding the $x$-1 constraints in Eq. (14) [30]. Therefore, the quality of non-dominant Pareto optimality calculated by the augmented $\varepsilon$-constraint method has been significantly improved compared with the original one.

\section{VALIDATION}

A numerical experiment is performed in order to validate the performance of the mathematical model and the two different solution methods. The structure of test parameters is adopted from a previous research work by Yu and Solvang [6] who employed a normalized weighted sum method to generate a set of ten Pareto optimality through the change of weight combination between cost efficiency and carbon emission. In this paper, the efficiency-versus-emission tradeoffs will be generated through augmented $\varepsilon$-constraint method, and the result is compared with the previous research.

The research work by $\mathrm{Yu}$ and Solvang [6] focuses on a reverse supply chain, so the current model is adjusted through bounding the demand constraint from the customer who becomes the starting points of a reverse supply chain. The numerical experiment aims at determining the configuration of a reverse supply chain including 10 suppliers for used products, 28 candidates for production plants with different technologies, 10 distribution centers and 5 customers. It is assumed all the used product supplied can be properly treated and all the recycled products can be sold in market, so the flow restrictions are re-formulated to adapt this change. The parameters used in the test are generated randomly within a given interval as described in Yu and Solvang [6].

The numerical example is a medium-sized problem and is programmed and resolved by Lingo optimization solver on a laptop computer with Intel Core i5-6200 2.4GHz CPU and 8 GB RAM. The decision-making problem of green supply chain design is first resolved with the normalized weighted sum method as given in the original research, and then the augmented $\varepsilon$-constraint method is used to generate a set of Pareto optimality.

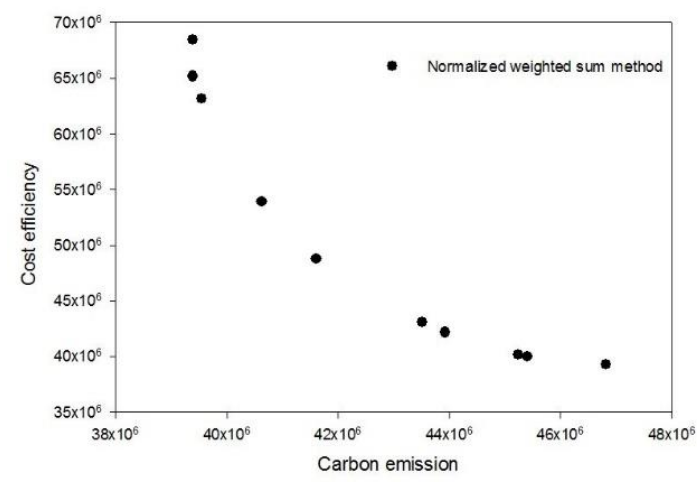

Figure 2. Optimal trade-offs solved by normalized weighted sum method.

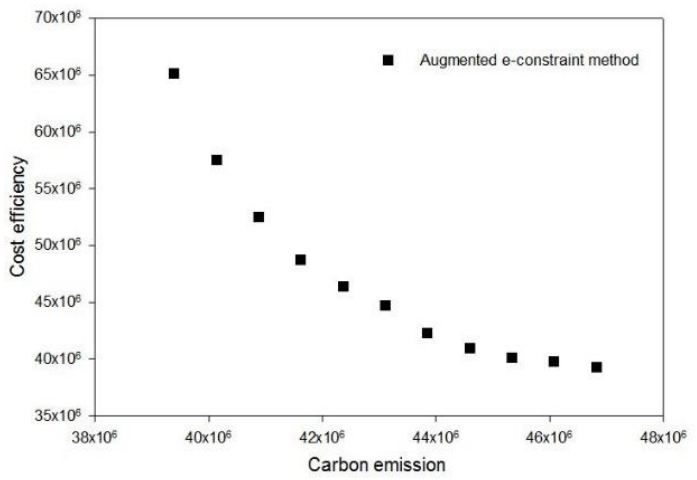

Figure 3. Optimal trade-offs solved by augmented $\varepsilon$-constraint method.

The optimal efficiency-versus-emission trade-offs solved by normalized weighted sum method and augmented $\varepsilon$ constraint method are given in Figures 2 and 3, respectively. Figure 4 shows the comparison of the Pareto optimality sets from the two solution methods.

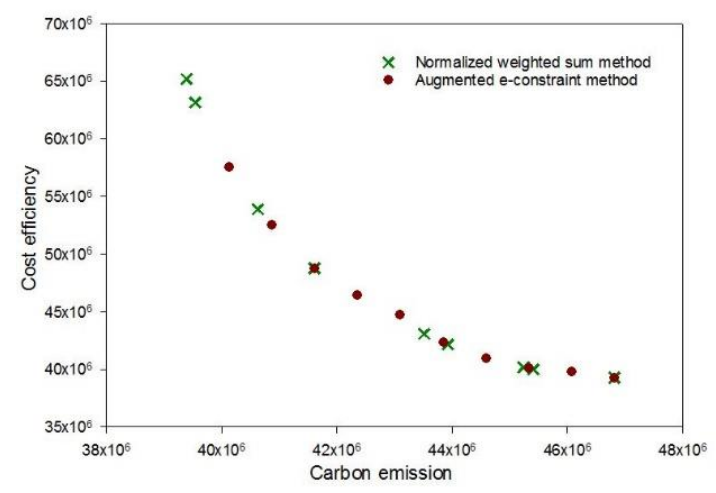

Figure 4. Comparison of the Pareto optimality.

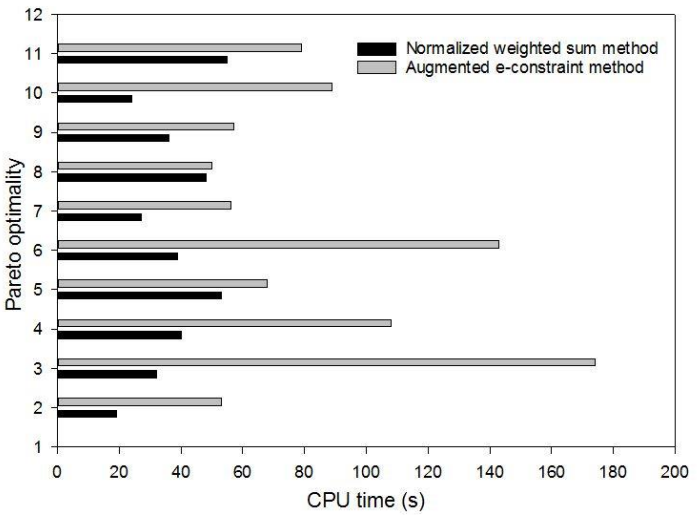

Figure 5. Comparison of the computational performance.

As shown in the figures, both solution method can generate a set of optimal efficiency-versus-emission tradeoffs in green supply chain design. The augmented $\varepsilon$-constraint method has a better performance than the weighted sum method in generating non-dominant optimal solutions, but this also leads to an increase on the computational time required as illustrated in Figure 5. 
This manuscript is a preprint version and final version is available on IEEE Xplore. Doi: 10.1109/ICITM.2018.8333934

\section{COUNCLUSION}

This paper investigates the efficiency-versus-emission trade-offs in green supply chain design. First, a bi-objective MILP model is formulated, and then two solutions methods are then introduced and tested. From the numerical validation, it is observed than the augmented $\varepsilon$-constraint method has a better performance in determining non-dominant set of Pareto optimality while weighted sum method requires less computational time. For future improvement of the current research, the focus may be given to the development of highperformance algorithm for large-scale integer programming problems in green supply chain design.

\section{ACKNOWLEDGMENT}

The authors would like to thank the support for this research from TARGET project financed by Northern Periphery and Arctic Programme (NPA).

\section{REFERENCES}

[1] S. Chopra and P. Meindl, "Supply chain management. Strategy, planning \& operation," Das summa summarum des management, pp. 265-275, 2007.

[2] M. S. Pishvaee, M. Rabbani, and S. A. Torabi, "A robust optimization approach to closed-loop supply chain network design under uncertainty," Applied Mathematical Modelling, vol. 35, no. 2, pp. 637649, 2011.

[3] M. T. Melo, S. Nickel, and F. Saldanha-Da-Gama, "Facility location and supply chain management-A review," European journal of operational research, vol. 196, no. 2, pp. 401-412, 2009.

[4] F. Wang, X. Lai, and N. Shi, "A multi-objective optimization for green supply chain network design," Decision Support Systems, vol. 51, no. 2, pp. 262-269, 2011.

[5] P. Ahi and C. Searcy, "A comparative literature analysis of definitions for green and sustainable supply chain management," Journal of Cleaner Production, vol. 52, pp. 329-341, 2013.

[6] H. Yu and W. D. Solvang, "A general reverse logistics network design model for product reuse and recycling with environmental considerations," The International Journal of Advanced Manufacturing Technology, vol. 87, no. 9-12, pp. 2693-2711, 2016.

[7] H. Yu and W. D. Solvang, "A carbon-constrained stochastic optimization model with augmented multi-criteria scenario-based riskaverse solution for reverse logistics network design under uncertainty," Journal of Cleaner Production, vol. 164, pp. 1248-1267, 2017.

[8] Y. Zhou, D.-C. Gong, B. Huang, and B. A. Peters, "The impacts of carbon tariff on green supply chain design," IEEE Transactions on Automation Science and Engineering, vol. 14, no. 3, pp. 1542-1555, 2017.

[9] S. Seuring and M. Müller, "From a literature review to a conceptual framework for sustainable supply chain management," Journal of cleaner production, vol. 16, no. 15, pp. 1699-1710, 2008.

[10] M. Brandenburg, K. Govindan, J. Sarkis, and S. Seuring, "Quantitative models for sustainable supply chain management: Developments and directions," European Journal of Operational Research, vol. 233, no. 2, pp. 299-312, 2014.

[11] S. Seuring, "A review of modeling approaches for sustainable supply chain management," Decision support systems, vol. 54, no. 4, pp. 15131520, 2013.

[12] M. Eskandarpour, P. Dejax, J. Miemczyk, and O. Péton, "Sustainable supply chain network design: an optimization-oriented review," Omega, vol. 54, pp. 11-32, 2015.

[13] K. Boonsothonsatit, S. Kara, S. Ibbotson, and B. Kayis, "Development of a Generic decision support system based on multi-Objective Optimisation for Green supply chain network design (GOOG),"
Journal of Manufacturing Technology Management, vol. 26, no. 7, pp. 1069-1084, 2015.

[14] S. Coskun, L. Ozgur, O. Polat, and A. Gungor, "A model proposal for green supply chain network design based on consumer segmentation," Journal of Cleaner Production, vol. 110, pp. 149-157, 2016.

[15] A. Diabat, R. Khodaverdi, and L. Olfat, "An exploration of green supply chain practices and performances in an automotive industry," The International Journal of Advanced Manufacturing Technology, vol. 68, no. 1-4, pp. 949-961, 2013.

[16] A. Alshamsi and A. Diabat, "A Genetic Algorithm for Reverse Logistics network design: A case study from the GCC," Journal of Cleaner Production, vol. 151, pp. 652-669, 2017.

[17] C. Miret, P. Chazara, L. Montastruc, S. Negny, and S. Domenech, "Design of bioethanol green supply chain: Comparison between first and second generation biomass concerning economic, environmental and social criteria," Computers \& Chemical Engineering, vol. 85, pp. 16-35, 2016.

[18] H. Yu, W. D. Solvang, and B. Solvang, "A Goal Programming Approach for Green Supply Chain Network Optimization," 2016.

[19] K. Govindan, A. Jafarian, and V. Nourbakhsh, "Bi-objective integrating sustainable order allocation and sustainable supply chain network strategic design with stochastic demand using a novel robust hybrid multi-objective metaheuristic," Computers \& Operations Research, vol. 62, pp. 112-130, 2015.

[20] L. Ameknassi, D. Ait-Kadi, and N. Rezg, "Integration of logistics outsourcing decisions in a green supply chain design: A stochastic multi-objective multi-period multi-product programming model," International Journal of Production Economics, vol. 182, pp. 165-184, 2016.

[21] K. Govindan, A. Jafarian, R. Khodaverdi, and K. Devika, "Twoechelon multiple-vehicle location-routing problem with time windows for optimization of sustainable supply chain network of perishable food," International Journal of Production Economics, vol. 152, pp. 9$28,2014$.

[22] M. Feitó-Cespón, W. Sarache, F. Piedra-Jimenez, and R. CespónCastro, "Redesign of a sustainable reverse supply chain under uncertainty: A case study," Journal of Cleaner Production, vol. 151, pp. 206-217, 2017.

[23] H. Yu and W. Solvang, "A Stochastic Programming Approach with Improved Multi-Criteria Scenario-Based Solution Method for Sustainable Reverse Logistics Design of Waste Electrical and Electronic Equipment (WEEE)," Sustainability, vol. 8, no. 12, p. 1331, 2016

[24] M.-J. Yao and H.-W. Hsu, "A new spanning tree-based genetic algorithm for the design of multi-stage supply chain networks with nonlinear transportation costs," Optimization and Engineering, vol. 10, no. 2, pp. 219-237, 2009.

[25] M. Sakawa, H. Yano, I. Nishizaki, and I. Nishizaki, Linear and multiobjective programming with fuzzy stochastic extensions. Springer, 2013.

[26] H. A. Eiselt and C.-L. Sandblom, Operations research: a model-based approach. Springer Science \& Business Media, 2012.

[27] H. Yu and W. D. Solvang, "An improved multi-objective programming with augmented $\varepsilon$-constraint method for hazardous waste locationrouting problems," International journal of environmental research and public health, vol. 13, no. 6, p. 548, 2016.

[28] I. Das and J. E. Dennis, "A closer look at drawbacks of minimizing weighted sums of objectives for Pareto set generation in multicriteria optimization problems," Structural and multidisciplinary optimization, vol. 14 , no. 1, pp. 63-69, 1997.

[29] G. Mavrotas, "Effective implementation of the $\varepsilon$-constraint method in multi-objective mathematical programming problems," Applied mathematics and computation, vol. 213, no. 2, pp. 455-465, 2009.

[30] J. Clímaco and J. Craveirinha, "Multiple criteria decision analysisstate of the art surveys," ed: New York: Springer, 2005, pp. 899-951. 